\title{
EDITORIAL
}

\section{The definition of a hemodynamically significant ductus}

\section{arteriosus}

Pediatric Research (2019) 85:740-741; https://doi.org/10.1038/s41390019-0342-7

A cause and effect relationship between a hemodynamically significant ductus arteriosus (DA) in premature infants and important short- and long-term morbidities remains unproven. Over 60 randomized controlled trials (RCTs) of patent DA (PDA) treatment have failed to demonstrate a reduction in important neonatal adverse outcomes, including intraventricular hemorrhage (IVH), necrotizing enterocolitis (NEC), chronic lung disease (CLD), death or neurodisability. ${ }^{1}$ Although the PDA is a plausible trigger for many of these complications, consensus is elusive with the recognition that causality has not been proven from the RCTs and the PDA may just be a common occurrence in this population. There are several reasons for the failure of RCTs to demonstrate an effect: (a) contamination of the control arm with open label PDA treatment; (b) difficulty in achieving PDA closure in a large number of infants in the treatment arm due to poor drug efficacy or inadequate drug delivery; ${ }^{2}$ and (c) sole focus on long-term neurodevelopment impairments with failure to recognize other significant pre- and post-discharge factors that can mitigate treatment effect (in either direction). However, the most important reason for lack of effect in those trials may lie in the heterogeneity and lack of clarity in characterizing the hemodynamic significance of the DA, determining the optimal treatment time, and identifying the "at-risk" population most likely to benefit from closure. Many studies have utilized arbitrary cutoffs of clinical and echocardiography parameters, unrelated to important short- and long-term outcomes, with an over-reliance on PDA diameter to determine significance. ${ }^{3}$

The concept of hemodynamic significance represents a complex interaction between several intrinsic and extrinsic factors, and quantification of this principle is a challenge in neonatal medicine. In order to characterize hemodynamic significance, several elements need to be considered: (1) PDA shunt volume assessment and its impact on the systemic and pulmonary circulations; ${ }^{4}$ (2) myocardial function evaluation, especially in considering how the heart handles the increased preload in the setting of potential myocardial ischemia secondary to impaired coronary artery perfusion; ${ }^{5}$ (3) antenatal and perinatal characteristics that can act as effect modifiers to either mitigate or exacerbate potential detrimental consequences of a shunt. ${ }^{6}$ Defining hemodynamic significance requires a thorough appraisal of all of these factors.

There is an increasing realization that an accurate estimation of shunt volume, rather than intermittent and discreet measurements of ductal diameter, provides a more holistic assessment of the hemodynamic impact of a PDA. ${ }^{7}$ Flow across the PDA is governed by Poiseuille's Law which states that: "At a constant driving pressure [the pressure gradients across the PDA], the flow rate of liquid through a tube is directly proportional to the fourth power of the radius of the tube and inversely proportional to the length of the tube and viscosity of the fluid. ${ }^{\text {" }}$ "In the setting of a PDA, the pressure gradients across the vessel, the length and diameter of the PDA and blood viscosity all constantly change over the first few days following birth. ${ }^{9}$ This makes direct determination of shunt volume extremely challenging. Echocardiography can be utilized to diagnose more than just the presence of a PDA, but also to estimate shunt volume by measuring surrogate echocardiographic markers of pulmonary over-circulation and systemic hypoperfusion. ${ }^{8}$ For example, a drop in celiac or mesenteric artery blood flow in the presence of PDA, despite a rising LV output (LVO), coupled with aortic run off all provide measures of systemic hypoperfusion. ${ }^{10}$ The left atrial-toaortic ratio (LA/Ao), the early passive filling to late active phase ratio $(E / A)$, the interval between closure of the aortic valve and mitral valve (isovolumic relaxation time, IVRT), and LVO are quantifiable indices of pulmonary over-circulation that can aid the delineation of hemodynamic significance of the DA. ${ }^{10}$ Although there are important considerations with each marker (e.g., pseudonormalization of some indices from offloading of the left atrium with a patent foramen ovale or atrial septal defect), ${ }^{11}$ recent evidence has demonstrated no difference between the measures in the presence of a large $(>2 \mathrm{~mm})$ atrial level shunt. ${ }^{7}$

Myocardial function is another important component of hemodynamic significance, but has often been forgotten in the assessment of a PDA and warrants further attention. Although much of the morbidity and mortality is related to the effects of the PDA on the systemic and pulmonary circulations, cardiac function is still an important driver in disease. The premature myocardium is characterized by systolic and diastolic dysfunction due to an inefficient contractile apparatus, a lack of compliant elastic and a preponderance of stiff fibrous tissue. ${ }^{12,13}$ In particular, diastolic dysfunction in the setting of increased preload can exacerbate the adverse consequences of increased pulmonary blood flow. Recent evidence from studies in premature infants has demonstrated the importance of left ventricular (LV) diastolic function in maintaining right ventricular (RV)-pulmonary artery (PA) coupling: an increase in afterload, results in an increase in myocardial contractility up to a certain threshold, beyond which any further increase in afterload results in compromised contractility (decoupling). The presence of LV diastolic compromise results in a reduction of this decoupling threshold. RV function cannot increase in the face of increased afterload in the presence of LV diastolic dysfunction due to pulmonary venous hypertension. ${ }^{14}$ This can have significant clinical implications with a recent study illustrating a relationship between diastolic dysfunction and the need for ventilation and occurrence of pulmonary hemorrhage in premature infants $<29$ weeks. $^{15}$ This relationship was independent of important confounders such as gestational age and antenatal steroids. ${ }^{15}$ In the setting of a PDA, decoupling could be further exacerbated due to the inability of the stiff LV to accommodate an increasing pulmonary venous return. Recent evidence also suggests that infants with lower diastolic function assessed using tissue Doppler imaging are more likely to suffer from PDA-related morbidities such as CLD. ${ }^{11}$ This highlights the importance of considering LV diastolic function in the definition of hemodynamic significance. ${ }^{11}$ 
The inclusion of important antenatal and perinatal clinical factors should also be an important part in characterizing hemodynamic significance. ${ }^{6}$ Lower gestational ages, growth restriction, lack of antenatal steroids, and other adverse perinatal events may also play a role in exacerbating the detrimental effects of PDA shunting. Therefore, a definition of a hemodynamically significant DA must not only include echocardiographic surrogate markers of shunt volume and a comprehensive assessment of LV diastolic function, but must also incorporate important clinical characteristics. Any such definition should also be prospectively evaluated to determine its ability to accurately predict ductalrelated morbidities. ${ }^{11}$ This will facilitate a more accurate identification of high-risk infants who are likely to benefit from treatment, thereby potentially maximizing the therapeutic benefit of PDA treatment.

There is recent evidence that suggests this approach provides an accurate predictive definition of hemodynamic significance. In a recent multicenter prospective observational study, EL-Khuffash et al. studied 141 infants at a mean \pm SD gestation of $26 \pm$ 1.4 weeks and performed a comprehensive echocardiogram between 36 and $48 \mathrm{~h}$ of life to assess whether a PDA severity score (PDAsc) incorporating markers of pulmonary over-circulation, left ventricular (LV) diastolic function, and clinical characteristics can predict chronic lung disease or death before discharge (CLD/Death). Using univariate analysis and multiple logistic regression, following five parameters that were independently associated with CLD/Death were identified: gestation at birth (an important clinical parameter), PDA diameter and flow velocity, left ventricular output (a marker of shunt volume and pulmonary overcirculation), and LV a' wave (a measure of diastolic function). The PDAsc had a range from 0 (low risk) to 13 (high risk). Infants who developed CLD/Death had a higher score than those who did not [7.3 (1.8) vs. $3.8(2.0), p<0.001]$. A cutoff PDAsc of 5.0 had an area under the curve (AUC) of 0.92 (95\% Cl 0.86-0.97, $p<0.001)$ for the ability to predict CLD/Death. The PDAsc cutoff of 5.0 had positive and negative predictive values of 92 and $82 \%{ }^{11}$

The decision to augment closure of a hemodynamically significant DA with medical or surgical mediation is beyond the scope of this editorial, but a similar comprehensive approach to characterizing a PDA might aid in the selection process for intervention, when warranted. ${ }^{11}$ Assessment of the hemodynamic significance of the DA with echocardiography has now become the standard of care in neonatal intensive care units ${ }^{16}$ with a growing recognition that it can provide hemodynamic information that either complements what is clinically suspected or delivers novel physiologic insight ${ }^{17}$ The recognized limitations of clinical and laboratory measures of cardiac output, systemic blood flow, and end-organ perfusion support the need for an extensive approach to the monitoring of the neonatal cardiovascular system that will provide the most complete and accurate picture of the cause of hemodynamic instability, inform decisionmaking, and offer a possible therapeutic approach. Intervention trials relying on simplistic definitions of hemodynamic significance, such as PDA diameter in isolation, are no longer adequate. The comprehensive appraisal of PDA importance outlined above can be used to define hemodynamic significance and identify high-risk infants for enrollment in modern era RCTs of PDA treatment.

\section{ADDITIONAL INFORMATION}

Competing interests: The authors declare no competing interests.

Publisher's note: Springer Nature remains neutral with regard to jurisdictional claims in published maps and institutional affiliations.

Afif El-Khuffash ${ }^{1}$, Philip T. Levy ${ }^{2}$, Matthias Gorenflo ${ }^{3}$ and Ivan D. Frantz III

${ }^{1}$ Department of Neonatology, The Rotunda Hospital and School of Medicine, Royal College of Surgeons in Ireland, Dublin, Ireland;

${ }^{2}$ Division of Newborn Medicine, Boston Children's Hospital and Department of Pediatrics, Harvard Medical School, Boston, MA, USA; ${ }^{3}$ Department of Pediatric Cardiology and Congenital Heart Diseases, University Hospital Heidelberg, Heidelberg, Germany and ${ }^{4}$ Department of Neonatology, Beth Israel Deaconess Medical Center and Department of Pediatrics, Harvard Medical School, Boston, MA,

Correspondence: Philip T. Levy (philip.levy@childrens.harvard.edu)

\section{REFERENCES}

1. Benitz, W. E. Treatment of persistent patent ductus arteriosus in preterm infants: time to accept the null hypothesis? J. Perinatol. 30, 241-252 (2010).

2. Bussmann, N. \& El-Khuffash, A. What is the most efficacious pharmacological therapy for patent ductus arteriosus closure in premature infants? Ir. Med J. 111, 816 (2018).

3. Zonnenberg, I. \& deWaal, K. The definition of a haemodynamic significant duct in randomized controlled trials: a systematic literature review. Acta Paediatr. 101, 247-251 (2012)

4. McNamara, P. J. \& Sehgal, A. Towards rational management of the patent ductus arteriosus: the need for disease staging. Arch. Dis. Child Fetal Neonatal Ed. 92, F424-F427 (2007).

5. Sehgal, A. \& McNamara, P. J. Coronary artery perfusion and myocardial performance after patent ductus arteriosus ligation. J. Thorac. Cardiovas Surg. 143, 1271-1278 (2012).

6. Kluckow, M. \& Lemmers, P. Hemodynamic assessment of the patent ductus arteriosus_Beyond ultrasound. Semin Fetal Neonatal Med 23, 239-244 (2018).

7. de Freitas Martins, F., Ibarra Rios, D. \& F Resende, M. H. Patent ductus arteriosus size to echocardiographic markers of shunt volume. J. Pediatr. 202, 50-55 (2018).

8. van Laere, D. et al. Application of NPE in the assessment of a patent ductus arteriosus. Pediatr. Res 84, 46-56 (2018).

9. Smith, A., McNamara, P. J. \& El-Khuffash, A. Non-pharmacological management of a hemodynamically significant patent ductus arteriosus. Semin Fetal Neonatal Med 23, 245-249 (2018).

10. El Khuffash, A., Jain, A. \& Mcnamara, P. J. Ligation of the patent ductus arteriosus in preterm infants: understanding the physiology. J. Pediatr. 162, 1100-1106 (2013).

11. El-Khuffash, A. et al. A patent ductus arteriosus severity score predicts chronic lung disease or death before discharge. J. Pediatr. 167, 1354-1361 (2015).

12. Noori, S. \& Seri, I. Pathophysiology of newborn hypotension outside the transitional period. Early Hum. Dev. 81, 399-404 (2005).

13. Fanaroff, J. M. \& Fanaroff, A. A. Blood pressure disorders in the neonate: hypotension and hypertension. Semin Fetal Neonatal Med 11, 174-181 (2006).

14. Bussmann, N. et al. Left ventricular diastolic function influences right ventricular pulmonary vascular coupling in premature infants. Early Hum. Dev. 128, 35-40 (2018).

15. Bussmann, N. et al. Early diastolic dysfunction and respiratory morbidity in premature infants: an observational study. J. Perinatol. 38, 1205-1211 (2018).

16. El-Khuffash, A. \& McNamara, P. J. Hemodynamic assessment and monitoring of premature infants. Clin. Perinatol. 44, 377-393 (2017).

17. EL-Khuffash, A. \& McNamara, P. J. Neonatologist-performed functional echocardiography in the neonatal intensive care unit. Semin. Fetal Neonatal Med. 16, 50-56 (2011). 